\title{
Study on edge cracking of titanium cold rolled sheet
}

\author{
Hyunseok lee, Miseon Choi
}

Research Institute of Industrial Science and Technology (RIST), 67, Cheaongam-ro, Nam-gu, Pohang-si, Gyeongsangbuk-do, Korea

\begin{abstract}
$\underline{\text { Abstract }}$
In this study, to understand oxygen effects on workability of titanium, edge cracking and microstructure changes with the oxygen content variations in the cold rolled sheets were observed carefully and discussed. The higher oxygen content of titanium sheet, the more the edge cracks occurred after cold rolling deformation. While the specimen containing low oxygen became fine grained structures after the cold rolling, the other high oxygen sheets consisted of relatively coarse and elongated grains. The fine-grained structures was assumed to come from an active twinning behavior, meanwhile the other's elongated structures were due to the twinning suppression by increasing oxygen contents. In addition, distinct shear bands were appeared in the high oxygen sheets after cold rolling. The high oxygen contents may multiply dislocation density by suppression of twinning and promoting dislocation slips. The increased dislocations can also produce a concentration of strain, slip bands thus appears during the cold rolling deformation. These slip bands cause the edge cracking in the higher oxygen sheets.
\end{abstract}

\section{Introduction}

Titanium and its alloys are used for a wide variety of applications thanks to their attractive properties which are corrosion resistance, low density and high strength. Especially, pure titanium, which has the outstanding resistance for corrosion, brings out to the most optimum material for the plate or pipe type heat exchanger, desalination facilities, chemical or petrochemical processing equipment, and so on[1].

Pure titanium is usually used as the commercial pure (CP) composition, which is intentionally adding impurities to control properties and microstructures. The mechanical properties of titanium strongly depends on interstitial elements such as oxygen[2]. Therefore, the oxygen content has to be carefully controlled to obtain optimal properties. The solid solution hardening, which is the most common hardening mechanism of titanium, is mainly implemented by the oxygen effect[3]. Anderson et al. noted that the oxygen content in titanium can show more lattice strain in the c direction than in the a direction[4]. Thus, the $\mathrm{c} / \mathrm{a}$ ratio of hcp titanium changes and the restricted dislocation motion affects the hardening of the titanium[5,6].

A cold rolling is the most common process for producing plate or pipe shape products of the CP titanium. Edge cracking, which often occurs during rolling with low workability material, significantly reduces yield and quality in the sheet production process. In the present study, the relationship between the oxygen content in pure titanium and the edge cracking during cold rolling was investigated.

\begin{tabular}{|c|c|c|c|c|c|}
\hline & $\mathrm{Fe}$ & $\mathrm{O}$ & $\mathrm{C}$ & $\mathrm{N}$ & (Weight\%) \\
\hline CP1 & 0.03 & 0.05 & 0.006 & 0.003 & 0.0001 \\
\hline CP3 & 0.13 & 0.20 & 0.010 & 0.004 & 0.0032 \\
\hline CP4 & 0.15 & 0.32 & 0.010 & 0.004 & 0.0017 \\
\hline
\end{tabular}

Table 1. Chemical composition of the titanium sheets used in this study
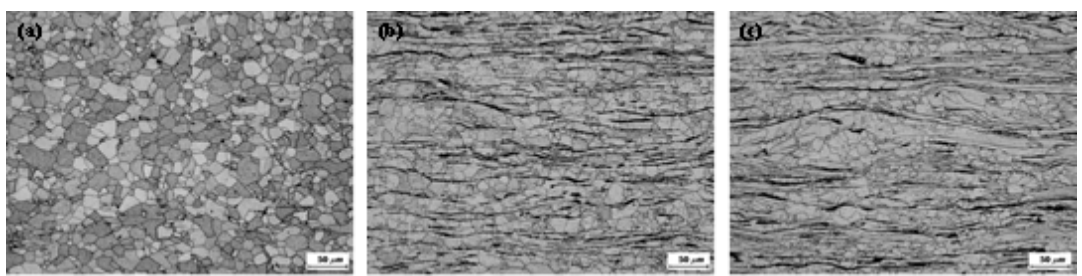

Figure 1. Optical micrographs of the hot rolled specimens (a)CP1, (b)CP2 and (c)CP3

\section{Material and experiments}

Three kinds of commercially pure titanium sheets were used in this experiment. Whereas they are hot rolled plate with the same thicknesses of 3.6 mm, their compositions are different each other as shown in Table 1 . The specimen, named as CP1, has the lowest oxygen and iron contents, while the CP3 and CP4 have the middle and highest contents, respectively. Figure 1 shows the microstructures of each hot rolled specimens. Although CP1 is almost composed of singe alpha phase, $\mathrm{CP} 3$ and CP4 are showing dispersed beta phases in alpha phase. The iron has a very low solubility in the alpha phase and it therefore is rejected and then forms the beta phase, either during solidification or subsequent cooling[7].

A Specimen for cold rolling, which was made from each hot rolled plate, had thickness, width and length of $3.6 \mathrm{~mm}, 100 \mathrm{~mm}$ and $250 \mathrm{~mm}$, respectively. Cold rolling tests was carried out using an laboratory rolling mill. The initiation and final thickness of the specimen were $3.6 \mathrm{~mm}$ and $1.0 \mathrm{~mm}$, thus the total reduction rate became $72 \%$. Meanwhile, each reduction rate per pass was less than $10 \%$ and silicon rolling lubricant was used.

Edge cracking and microstructure changes with the oxygen content variations in the cold rolled sheets were observed carefully and discussed.

(C) The Authors, published by EDP Sciences. This is an open access article distributed under the terms of the Creative Commons Attribution License 4.0 (http://creativecommons.org/licenses/by/4.0/). 


\section{$\underline{\text { Results and Discussion }}$}

Figure 2 is exhibiting edge cracks of the cold rolled specimens, CP1, CP2 and CP3. While any distinct crack is not found in CP1 sheet, which is the lowest oxygen content, Lots of cracks were appeared on the edge of CP3 and CP4 sheets. Figure 3 is displaying the change in the number of edge cracks per 100 mm length depending on the oxygen content. The higher oxygen content of the cold rolled sheet, the more the edge cracks occurred. Figure 4 shows the optical microstructures in RD-ND cross sectional planes of the cold rolled sheets. It can be seen in Figure 4 (b) and (c) that the edge cracks in CP3 and CP4 had similarly occurred along about 45 degrees from the surface. The high magnification (x1000) pictures of the same specimens with Figure 4 are displayed in Figure 5. While the cold rolled sheet from CP1 having the low oxygen content is fine grained structures (Figure 5 (a)), the other sheets of the higher oxygen contents are showing relatively coarse and elongated grains (Figure 5 (b) and (c)).
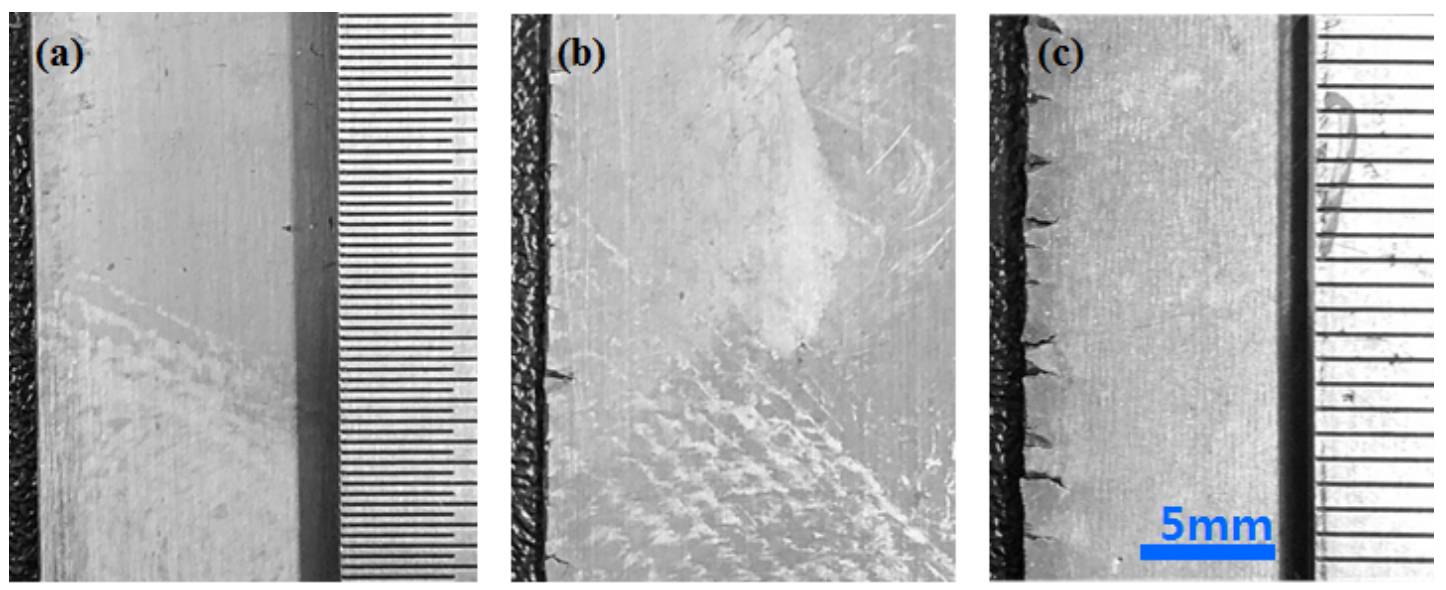

Figure 2. Edge cracks of the cold rolled sheets (a)CP1, (b)CP2 and (c)CP3

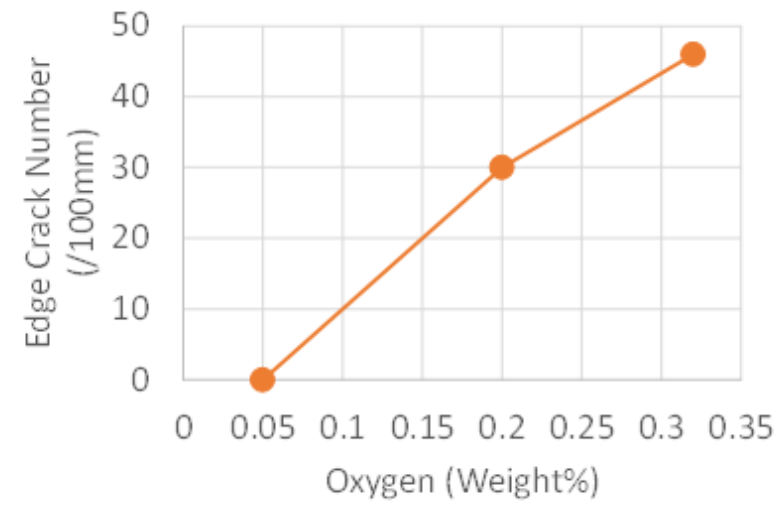

Figure 3. Variations in the number of edge cracks (/100 mm length)

According to the past similar studies[8 10], the various microstructures can be developed by the oxygen contents which significantly affect the operative mode during a deformation. Kang et al.[9] reported that as the oxygen content increases in pure titanium, the deformation twinning becomes suppressed and slip deformation is promoted. The suppression of twinning by oxygen can be considered to be due to the two factors: firstly, the twinning deformation is interfered by increasing the value of c/a, an axial ratio in the hcp structures as seen. As for second reason, atomic shuffles are considered to be an important factor in producing at twinned structure. Meanwhile, Microstructure characteristics of a heavy cold rolled commercially pure titanium sheet were investigated and discussed by $\mathrm{n}$. Bozzol et al.[8]. The 80\% cold rolled microstructure of commercially pure titanium (grade 2) is constituted by two types of local structures: the main 85 volume \% of fine-subdivided structures by twinning and the rest 15 volume $\%$ of lamellar structures which is without twinning deformation. 

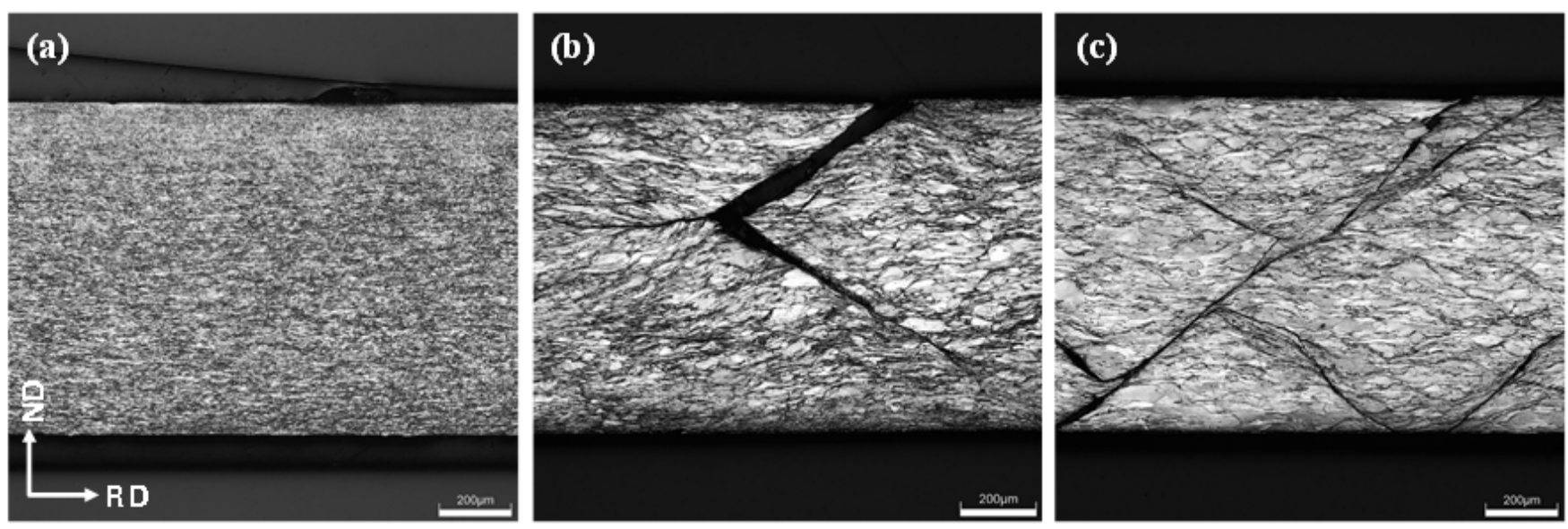

Figure 4. RDxND cross-sectional micrographs of the cold rolled specimens(x200 magnification) (a)CP1, (b)CP2 and (c)CP3
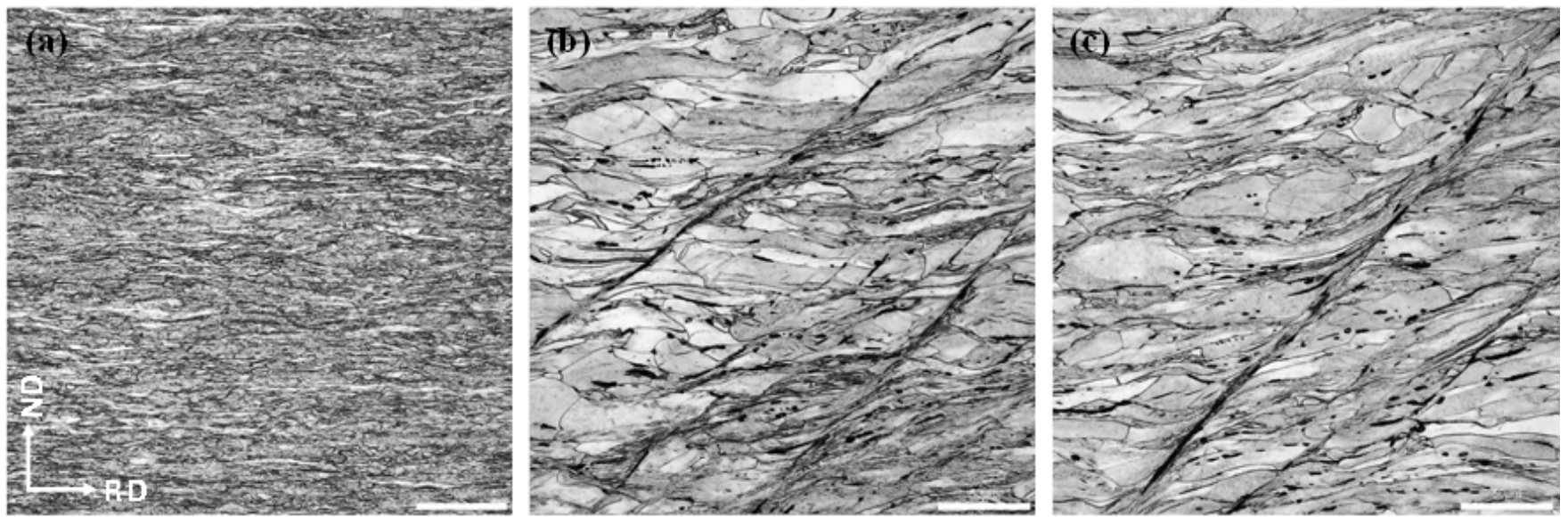

Figure 5. RDxND cross-sectional micrograph of the cold rolled specimens(x1000 magnification) (a)CP1, (b)CP2 and (c)CP3

In the present study, it is assumed that the fine-grained structures of CP1 is coming from an active twinning behavior, while the other's elongated structures are due to the twinning suppression by increasing oxygen contents. In addition, dissimilar from CP1, the cold rolled microstructures of CP3 and CP4 are markedly displaying shear bands as shown in Figure 5. Shear bands (adiabatic shear bands) occur in which the heat generated in localized bands which are unable to be dissipated because of the high strain rate and poor thermal properties of metals. Titanium and titanium alloys are known to be one of the most susceptible materials to adiabatic shear banding due to their low heat diffusivity properties[11 13]. Considering failures of metals mainly initiate at the boundaries of shear bands under high strain rate conditions, the edge cracking in the higher oxygen concentration titanium, as seen in Figure 4 (b) and (c), are assumed to be closely related with shear bands occurrences. In the previous paper, it was said that the dislocation density was increased as a substitute instead of twinning for deformation compatibility as increasing oxygen concentration in titanium. The increased number of dislocations, as a schematic illustration in Figure 6, may produce a concentration of strain, thereby reducing local elongation and potential causing fracture in the vicinity of the grain boundaries[9].

Based on the experimental results and previous studies, it can be assumed that the high oxygen concentration can multiply dislocation density by suppression of twinning and promoting dislocation slips. The increased number of dislocations may also produce a concentration of strain, eventually slip bands appears during the cold rolling deformation. The slip bands is the main reason for the edge cracking in the sheets.

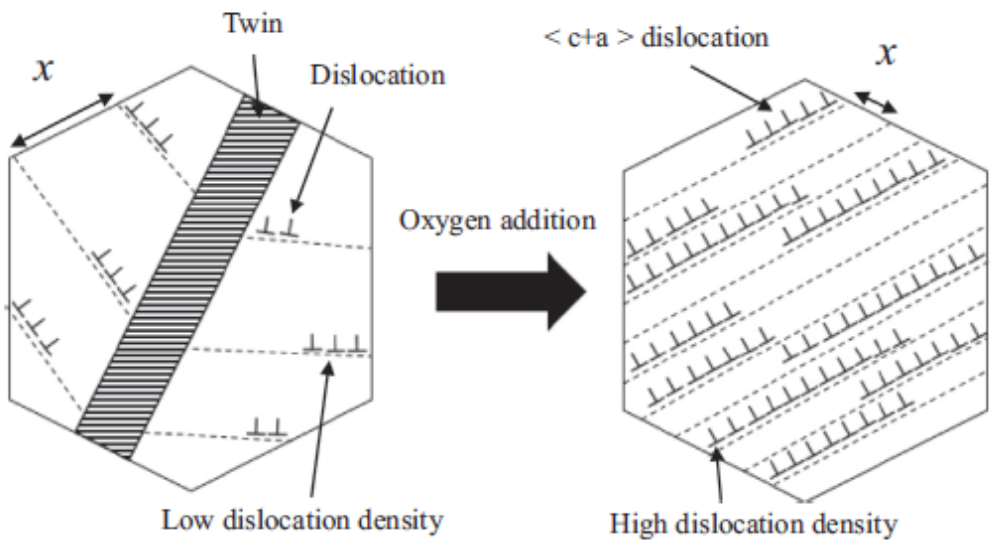

Figure 6. Schematic illustration of the deformation microstructure in Ti-O alloy[9] 


\section{Conclusion}

To understand the effect of oxygen on the workability of titanium, a cold rolling test was conducted with titanium sheet having various oxygen contents. The edge cracking of the cold rolled specimens were investigated and discussed as follows:

(1) Any edge crack was not found in CP1 sheet containing the lowest oxygen content, although many cracks were occurred on the edge of CP3 and CP4 having the higher oxygen contents after the cold rolling deformation.

(2) While the CP1 specimen became fine grained structures after the cold rolling, the other high oxygen composition sheets, CP3 and CP4, consisted of relatively coarse and elongated grains. The fine-grained structures of CP1 was assumed to come from an active twinning behavior, meanwhile the other's elongated structures were due to the twinning suppression by increasing oxygen contents. In addition, CP3 and CP4 were showing distinct shear bands in the cold rolled microstructures.

(3) The high oxygen contents, existing as interstitial element in titanium, may multiply dislocation density by suppression of twinning and promoting dislocation slips. The increased number of dislocations can also produce a concentration of strain, slip bands thus appears during the cold rolling deformation. These slip bands cause the edge cracking in the higher oxygen sheets.

\section{References}

[1] F .H. Froes, Titanium : Physical Metallurgy Processing and Applications (2015) 66

[2] D. J. Simbi and J. C. Scully, Mater. Lett. 26 (1996) 35

[3] J.-M. Oh, B.-G. Lee, S.-W. Cho, S.-W. Lee, G.-S. Choi, and J.-W. Lim, Met. Mater. Int., Vol. 17, No. 5 (2011) 733

[4] S. Anderson, B. Collen, V. Kuylenstierna, and A. Magneli, Acta. Chem. Scand. 11 (1957) 1641

[5] G. A. Sargent and H. Conrad, Scr. Metall. 6 (1972) 1099

[6] M. Dechamps, A. Quivy, G. Baur, and P. Lehr, Scr. Metall. 11 (1977) 941

[7] G. Lutjering, J. C. Williams, Titanium, 2nd ed. (2007) 181

[8] N. Bozzolo, N. Dewobroto, H. R. Wenk, F. Wagner, J Mater Sci (2007) 42 2405-2416

[9] Duck-soo Kang, Kwang-jin Lee, Eui-pyo Kwona, Toshihiro Tsuchiyama, Setsuo Takaki, Mater. Sci. \& Eng. A 632 (2015) 120

[10] Jong Woo Won, Chan Hee Park, Jaekeun Hong, Chong Soo Lee, Seong-Gu Hong, Scientific Reports (2019) 9:2009

[11] A.R Shahan, A.Karimi Taheri, Materials \& Design, Volume 14, Issue 4, (1993) 243

[12] J. Peirs, W. Tirry, B. Amin-Ahmadi, F. Coghe, P. Verleysen, L. Rabet, D. Schryversb, J. Degrieckam, Mater. characterization 75 (2013) 79

[13] Dengke Yang, Yang An, Pavel Cizek, Peter Hodgson, Mater. Sci. \& Eng. A 528 (2011) 3990 